stinaltrakt bzw. Blutdruckabfall auf. Die kumulativen Schwellendosen betrugen 10 bis $50 \mathrm{mg}$ Soja für subjektive Symptome und $454 \mathrm{mg}$ bis $50 \mathrm{~g}$ für objektive Symptome. Unter der Annahme einer Normalverteilung bedeutet dies, dass $1 \%$ aller Patienten mit einer Sojaallergie bereits auf $21 \mathrm{mg}$ Soja mit subjektiven und auf $37,2 \mathrm{mg}$ mit objektiven allergischen Symptomen reagieren. Das Profil der IgE-Reaktivität gegen die Soja- und Erdnussproteine war komplex und im Vergleich der Probanden sehr unterschiedlich, vor allem aber korrelierte es nicht mit der Schwere der allergischen Symptome.

Fazit: Die Schwellendosen, ab denen Sojaprotein bei entsprechend sensibilisierten Personen eine allergische Reaktion auslösen kann, liegen zwischen 10 und $50 \mathrm{mg}$ Soja für subjektive und
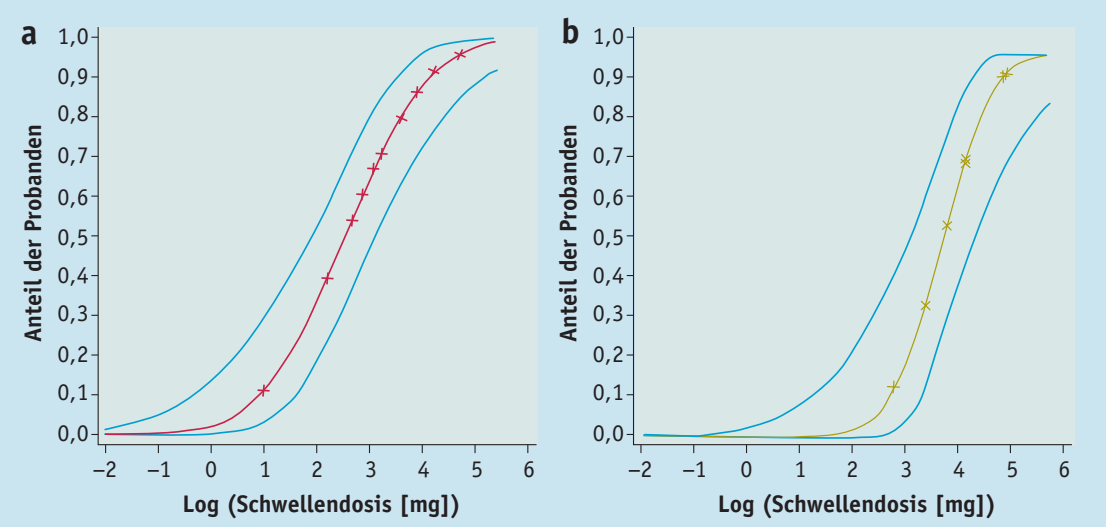

Kumulativer Anteil der Probanden mit subjektiven (a) oder mit objektiven Symptomen (b) in Abhängigkeit von den verabreichten Sojaprovokationsdosen (Kreuze)

zwischen $454 \mathrm{mg}$ und $50 \mathrm{~g}$ für objektive Symptome. Die Schwellendosis korreliert nicht mit der Schwere der Symptomatik.
Ballmer-Weber BK et al. Clinical characteristics of soybean allergy in Europe: a double-blind, placebo-controlled food challenge study. J Allergy Clin Immunol 2007; 119: 1489-96

\title{
Pektin schützt Allergene
}

\section{Proteine werden im sauren Milieu des Magens angegriffen und abgebaut. Damit Allergene ihre immunoge Wirkung entfalten können, müssen sie aber unbeschadet bis in den Darm gelangen. Bei Früchten scheinen dabei Pektine zu helfen.}

n Früchten sind verschiedene pflanzliche Polysaccharide wie Pektine enthalten. In Umgebung mit einem niedrigen $\mathrm{pH}-$ Wert bilden sie Hydrogele. $\mathrm{Ob}$ das dazu führt, dass Allergene unbeschadet den Magen passieren können, untersuchten serbische Forscher in vitro anhand eines Magensaftmodells sowie in vivo an gesunden Versuchspersonen.

Für die In-vitro-Untersuchungen wurden Fruchtextrakte von Kiwi, Banane, Apfel und Kirsche sowie Act c 2, das isolierte Majorallergen der Kiwi, verwendet. Es zeigte sich, dass Rohextrakte der verschiedenen Früchte widerstandsfähig gegenüber dem Pepsin des künstlichen Magensafts waren, nicht aber der aufbereitete Allergenextrakt, der bereits nach 15 Minuten vollständig verdaut war. Der Proteinschutz in den Rohextrakten war experimentell nicht durch einen hohen Gehalt an
Mono- und Disacchariden oder einen spezifischen Pepsininhibitor zu erklären, sondern schien eindeutig auf die sauren Polysaccharidketten des Pektins

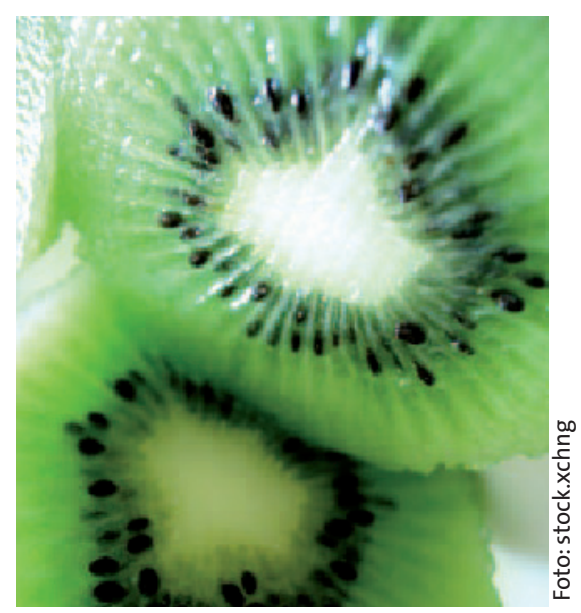

Pektine bewahren Act c 2, das Majorallergen der Kiwi, vor der Verdauung im Magen. zurückzuführen zu sein. Für diese Erklärung spricht auch, dass die Zugabe von Apfelpektin (1,5 oder 3\%) zum Kiwiallergenextrakt in der Lage war, das Allergen in vitro vor der Proteinverdauung zu schützen.

Bezüglich der Kiwi wurden die Befunde auch bei gesunden Probanden bestätigt: In deren Mageninhalt konnten eine Stunde nach Kiwiaufnahme sowohl intaktes Kiwiallergen Act c 2 als auch andere höhermolekulare Proteine entdeckt werden.

Die Autoren gehen davon aus, dass pektinreiche Früchte aufgrund des Matrixeffekts der sauren Polysaccharide die frühe Verdauung von Proteinen teilweise verhindern und damit für den Prozess der Sensibilisierung eine Rolle spielen.

Fazit: Pektine scheinen Allergene vor der Pepsinverdauung im Magen zu schützen. Pektinreiche Früchte könnten daher für die Entwicklung einer Sensibilisierung über das Einwirken intakter Allergene auf die Darmmukosa eine Rolle spielen.

Polovic $\mathbf{N}$ et al. A matrix effect in pectinrich fruits hampers digestion of allergen by pepsin in vivo and in vitro. Clin Exp Allergy 2007; 37: 764-71 